\title{
Manipulating neurons in an activity-dependent manner
}

Neurons that are activated in response to a particular stimulus or behavior can be labeled or manipulated with a new method.

Activated neurons typically express immediate-early genes such as Fos, a fact that has been used to detect and label these neurons. Fos-dependent tools are available to manipulate activated neurons. However, these tools may not recapitulate endogenous Fos expression faithfully, or they may not be very efficient.

"I have really always wanted to do the activity-dependent labeling and manipulation of the neurons," says Fan Wang from Duke University Medical Center. But because of her training as a geneticist, she quickly realized the limitations of existing tools. So she and her team set out to develop a versatile method that would work in many or all of the brain regions in mice and that would be useful for both labeling and manipulating neurons that were activated by a stimulus or behavior of interest.

Wang's approach was inspired by work on monosynaptic neural tracers, which are used to analyze connectivity in the brain. These tracers are based on a two-part system consisting of pseudotyped lentiviruses or rabies viruses and the TVA receptor. The viruses are coated with an EnvA envelope protein and encode a fluorescent marker, while the TVA receptor is exogenously expressed on neurons of interest and serves as a receptor for EnvA.

In this capturing activated neuronal ensembles (CANE) approach, TVA is expressed from the endogenous Fos locus, and thus it is only present in activated neurons. For improved temporal control, the TVA receptor is destabilized with a sequence that targets the protein for rapid degradation. The second component, the EnvA-pseudotyped virus, is injected into the brain region of interest. To reduce background noise, Wang and her colleagues used an EnvA mutant that has reduced affinity for the TVA receptor, thereby ensuring that only neurons with high Fos and TVA expression are infected by the pseudotyped virus. The researchers typically use pseudotyped lentiviruses that lead to the expression of the Cre recombinase in combination with Cre-dependent adenoassociated viruses that harbor fluorescent markers or with tools to manipulate neurons.

Getting all these components to work together to precisely and efficiently produce results was not a trivial feat. Wang says that it took almost six years to develop the technology. "For a while we had the specificity but we didn't have the very high efficiency that we needed," she said. The team eventually discovered that a high titer and purity of the lentivirus preparation was crucial for CANE to work. To achieve high titer and purity, they used a serum-free culture and concentrated the virus by centrifugation at low speed overnight.

Once the technology was established, Wang and her team used it to study neurons in the hypothalamus that are activated by social behaviors. They identified a subset of neurons in the mouse hypothalamus, which they named social-fear neurons, that are activated in an intruder who is attacked by an aggressive resident. These neurons are distinct from neurons that are located in the same area but activated by aggressive social behaviors. By driving channelrhodopsin 2 with the CANE technology to activate the social-fear neurons, the researchers could induce behaviors consistent with those of social fear. Conversely, inactivating the social-fear neurons with the CANE approach reduced social-fear behavior.

By combining a monosynaptic retrograde tracing approach with the CANE
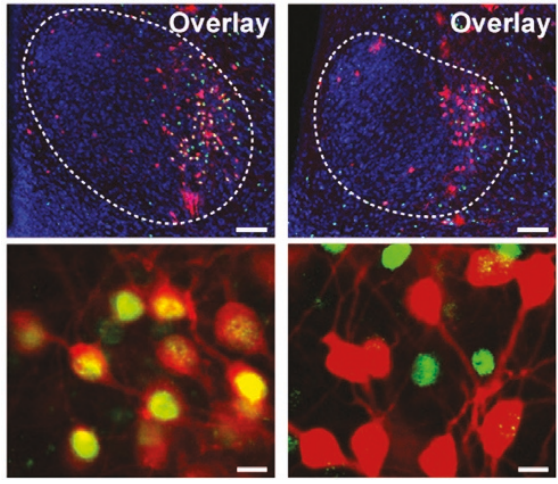

Repeated exposure to social fear activates the same neurons in the hypothalamus (left, red and green), while different neurons (right) are activated by social fear (red) and aggression (green). Figure reprinted from Sakurai et al. (2016) with permission from Elsevier.

technology, Wang and her colleagues identified the presynaptic inputs into the hypothalamic social-fear neurons. This allowed them to identify input neurons in several brain regions such as the nucleus accumbens, the lateral septum and others.

The main requirement for the CANE method is robust Fos expression in the neurons of interest. When Fos expression is too low, the cells are inefficiently labeled. Wang says that the CANE approach works not only in the context of social-fear behavior, but also in the pain circuit that her lab is working on. In addition, her collaborators have applied CANE to vocalization behavior, motor learning and olfaction, just to name a few areas. "It seems everywhere we apply it, it works," says Wang.

Nina Vogt

\section{RESEARCH PAPERS}

Sakurai, K. et al. Capturing and manipulating activated neuronal ensembles with CANE delineates a hypothalamic social-fear circuit. Neuron $\mathbf{9 2 ,}$ 739-753 (2016). 